\title{
Cutpoints in the conjunction of two graphs
}

\author{
By
}

Paul Hafner and Frank Harary

1. Definitions and examples. Let $G=(V, E)$ be a graph with vertex set $V$ and edge set $E$; similarly let $G_{1}=\left(V_{1}, E_{1}\right)$ and $G_{2}=\left(V_{2}, E_{2}\right)$. The conjunction $G=G_{1} \wedge G_{2}$ is defined by $V=V_{1} \times V_{2}$ and $\{u, v\}=\left\{\left(u_{1}, u_{2}\right),\left(v_{1}, v_{2}\right)\right\} \in E$ if and only if

$$
\left\{u_{1}, v_{1}\right\} \in E_{1} \text { and }\left\{u_{2}, v_{2}\right\} \in E_{2} \text {. }
$$

This binary operation on graphs was introduced by Weichsel [6] and later termed conjunction by Harary and Wilcox [4].

Weichsel proved that $G_{1} \wedge G_{2}$ is connected if and only if both $G_{1}$ and $G_{2}$ are connected and one of them contains an odd cycle. If both $G_{1}$ and $G_{2}$ are connected and bipartite, their conjunction $G$ consists of two connected components constructed as follows. Color both $V_{1}$ and $V_{2}$ red and green. Then one component of $G$ contains all vertices $\left(u_{1}, u_{2}\right)$ where $u_{1}, u_{2}$ have the same color and the other component contains the pairs of vertices of opposite color.

We now develop some definitions and examples leading to a criterion for $G=G_{1} \wedge G_{2}$ to be 2-connected, in other words, nonseparable or a block. Following the notation of [2], we write $C_{n}$ for the cycle of length $n$, and $P_{n}$ for the path with $n$ vertices which we now label $1,2,3, \ldots, n$ starting at one endpoint and moving to the other. If $u$ is a point of graph $H$ we will denote the points of $H \wedge P_{n}$ by $u 1, u 2$, etc. Terminology not defined here may be found in [2].

Weichsel remarked that the conjunction $K_{1, r} \wedge K_{1, s}$ of two stars has the two connected components $K_{1, r s}$ and $K_{r, 8}$. One acquires familiarity with the operation

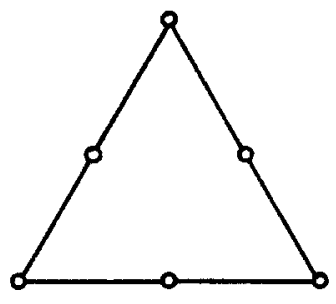

$C_{3} \wedge P_{2}$

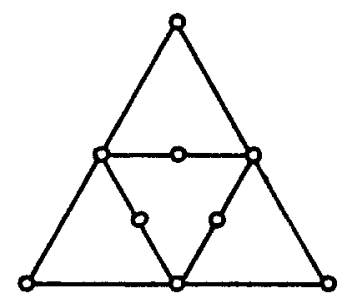

$C_{3} \wedge P_{3}$

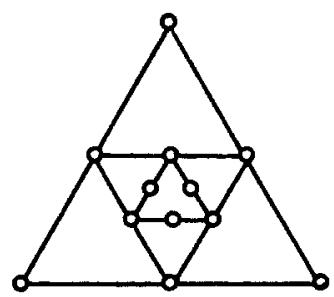

$C_{3} \wedge P_{4}$

Fig. 1. Three conjunctions of a triangle and a path 
by verifying that $C_{n} \wedge P_{2}=C_{2 n}$ if $n$ is odd and $H \wedge P_{2}=2 H$ if $H$ is bipartite; it is also instructive to construct $C_{n} \wedge K_{1, m}$. As another illustration Figure 1 shows $C_{3} \wedge P_{m}$ for $m=2,3,4$. One sees at once that the conjunction of a cycle and a path has no cutpoints (although it may be disconnected as $C_{2 n} \wedge P_{3}$ ).
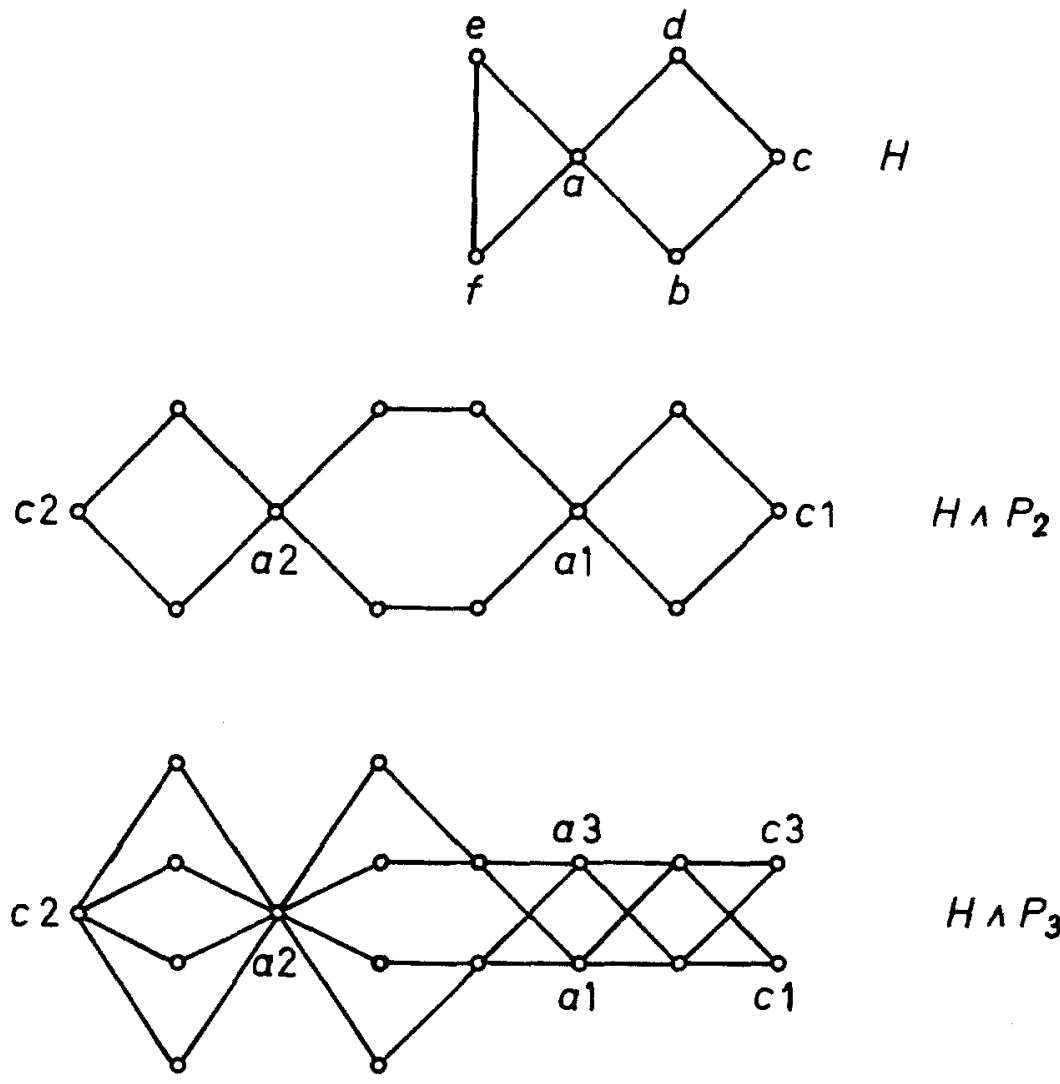

$H \wedge P_{3}$

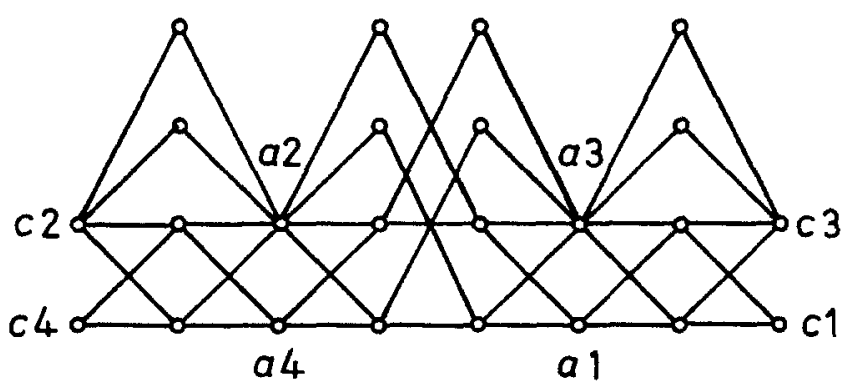

$H \wedge P_{4}$

Fig. 2. Three conjunctions of a graph with a path 
We will find that the typical behaviour regarding the occurrence of cutpoints in a conjunction is seen in the diagrams of Figure 2 which show $H \wedge P_{2}, H \wedge P_{3}, H \wedge P_{4}$ when $H=C_{3} \cdot C_{4}$ is the graph obtained by identifying a point of $C_{3}$ with a point of $C_{4}$.

If $u$ is a cutpoint of the connected graph $H$ then the removal of $u$ disconnects $H$, leaving a number of connected graphs $H_{1}, \ldots, H_{r}$. The subgraphs $F_{1}, F_{2}, \ldots, F_{r}$ of $H$ induced by $u$ and $H_{1}, u$ and $H_{2}, \ldots$ are called the branches of the cutpoint $u$. Note that $u$ is not a cutpoint of any $F_{i}$.

\section{A necessary condition.}

Proposition. Let $G_{1}$ and $G_{2}$ be connected graphs and assume $G=G_{1} \wedge G_{2}$ is connected. If $(x, y)$ is a cutpoint of $G$ then $x$ is a cutpoint of $G_{1}$ and $y$ is a cutpoint of $G_{2}$.

Proof. Denote by $F$ one of the branches of $(x, y)$ in $G$ and by $H$ the union of the remaining branches of $(x, y)$. Let $\left(a_{1}, b_{1}\right), \ldots,\left(a_{r}, b_{r}\right)$ be the vertices of $F$ adjacent to $(x, y)$ in $G$ and $\left(c_{1}, d_{1}\right), \ldots,\left(c_{s}, d_{s}\right)$ the remaining vertices adjacent to $(x, y)$ in $G$. We shall prove that in $G_{1}$ any path from $a_{i}$ to $c_{j}$ goes through $x$ provided $a_{i}$ is not terminal, and similarly in $G_{2}$. (If $a_{i}$ is terminal then obviously $x$ is a cutpoint of $G_{i}$.) So we assume that there exists a point $a_{i}^{\prime} \in V\left(G_{1}\right)$ adjacent to $a_{i}$ with $a_{i}^{\prime} \neq x$. Then $(x, y),\left(a_{i}, b_{i}\right),\left(a_{i}^{\prime}, y\right),\left(a_{i}, d_{j}\right)$ is a path in $G$ which avoids $(x, y)$ and hence $\left(a_{i}, d_{j}\right)$ $\in V(F)$; this proves that $a_{i} \neq c_{j}$ for all $j$. Now let $a_{i}, p_{1}, p_{2}, \ldots, p_{t}, c_{j}$ be a path in $G_{1}$ which avoids $x$. If $t$ is odd we get a path $\left(a_{i}, b_{i}\right),\left(p_{1}, y\right),\left(p_{2}, d_{j}\right), \ldots,\left(p_{t}, y\right)$, $\left(c_{j}, d_{j}\right)$ in $G$ which contradicts the fact that in $G$ the point $\left(c_{j}, d_{j}\right)$ can only be reached from $\left(a_{i}, b_{i}\right)$ via $(x, y)$. If $t$ is even then $G_{1}$ contains an odd $C: x, a_{i}, p_{1}, \ldots, p_{t}, c_{j}$. We now consider the path $b_{i}, y, b_{j}$ or $b_{i}, y$ in $G_{2}$ depending on whether $b_{i} \neq b_{j}$ or $b_{i}=b_{j}$ and denote it by $P_{3}$ or $P_{2}$ respectively. Clearly $C \wedge P_{3}$ and $C \wedge P_{2}$ have no cutpoints as seen earlier in Figure 1 . Hence there is a path in $G$ connecting $\left(a_{i}, b_{i}\right)$ and $\left(c_{j}, b_{j}\right)$ which avoids $(x, y)$. As this is a contradiction, it follows that every path in $G_{1}$ joining $a_{i}$ and $b_{j}$ meets $x$, i.e., $x$ is a cutpoint of $G_{1}$. If $b_{i}$ is not terminal (in which case $y$ is trivially a cutpoint) one finds that $y$ is a cutpoint of $G_{2}$ in the same fashion.

Now we know that if we want to find a cutpoint of $G$ it is sufficient to consider the pairs of cutpoints of $G_{1}$ and $G_{2}$. The following section shows, however, that this produces cutpoints only in rare cases.

\section{The searcity of eutpoints in $\mathbf{G}$.}

Lemma. Let $G_{1}$ be connected and not bipartite and assume $x$ is a cutpoint of $G_{1}$.

(a) $x 1$ is a cutpoint of $G_{1} \wedge P_{2}$ if and only if $x$ has a branch which is bipartite.

(b) $x 1$ and $x 3$ are not cutpoints of $G_{1} \wedge P_{3}$. Also $x 2$ is a cutpoint of $G_{1} \wedge P_{3}$ if and only if $x$ has a branch which is bipartite.

(c) $x 3$ is a cutpoint of $G_{1} \wedge P_{4}$ if and only if $x$ is adjacent to a terminal vertex.

Proof. Clearly (a) is implied by (b); also (a) follows from the construction given in Figures 1 and 2. 
We now prove (b). As 1 and 3 are not cutpoints of $P_{3}$, the Proposition implies that $x 1$ and $x 3$ cannot be cutpoints of $G_{1} \wedge P_{3}$. To study $x 2$, let $X_{1}, X_{2}, \ldots, X_{n}$ be the branches of $x$ in $G_{1}$ and recall that $x$ is not a cutpoint of any of them. If $X_{i}$ is bipartite then $X_{i} \wedge P_{3}$ has 2 connected components, one containing $x 1$ and $x 3$ and the other containing $x 2$. The removal of $x 2$ will disconnect the latter component from the rest of $G_{1} \wedge P_{3}$. If every branch $X_{i}$ contains an odd cycle then all $X_{i} \wedge P_{3}$ are connected and $x 2$ is not a cutpoint of any of them (since $x$ is not a cutpoint of any $\left.X_{i}\right)$. Now $G_{1} \wedge P_{3}$ can be obtained by identifying the vertices $x 1$ in $X_{1} \wedge P_{3}, X_{2} \wedge P_{3}$ etc., and the same with $x 2$ and $x 3$. When the vertex $x 2$ is removed the remainder of the $X_{i} \wedge P_{3}$ still hold together thanks to $x 1$ and $x 3$.

To prove (c), if $x$ is adjacent to the terminal vertex $u$ then $u 4$ is terminal and adjacent to $x 3$ in $G_{1} \wedge P_{4}$; hence $x 3$ is a cutpoint of $G_{1} \wedge P_{4}$. If on the other hand all vertices adjacent to $x$ are nonterminal we show that $x 3$ is not a cutpoint. Assume $u$ and $w$ are adjacent to $x$, and $v \neq x$ is adjacent to $u, z \neq x$ is adjacent to $w$. 'Typical paths across $x 3$ are

$$
\begin{aligned}
& u 2, x 3, u 4, \\
& u 2, x 3, w 2, \\
& u 2, x 3, w 4 .
\end{aligned}
$$

But one can always bypass $x 3$ as follows:

$$
\begin{aligned}
& u 2, v 3, u 4, \\
& u 2, x 1, w 2, \\
& u 2, x 1, w 2, z 3, w 4 .
\end{aligned}
$$

We may summarize these observations by saying that the connected conjunction $G=G_{1} \wedge G_{2}$ has no cutpoints provided $G_{1}$ has an odd cycle, every vertex of $G_{2}$ is contained in a path $P_{4}$, and not both $G_{1}$ and $G_{2}$ contain terminal vertices. The latter condition is a trivial one and is therefore incorporated into the hypothesis of the following criterion.

Theorem. Let $G_{1}$ and $G_{2}$ be both connected, not both bipartite, and not both containing terminal vertices. Then $G=G_{1} \wedge G_{2}$ has a cutpoint if and only if one of $G_{1}$ and $G_{2}$ is a star and the other has a bipartite block.

Proof. Assume $G$ has a cutpoint and $G_{1}$ contains an odd cycle. The remark just before the theorem excludes any possibility apart from $G_{2}$ being a star $K_{1, n}, n \geqq 1$ (since $G_{2}$ must have a cutpoint and no $P_{4}$ ). The Lemma then forces the condition that $G_{1}$ has a bipartite block, as $G_{1}$ must contain a cutpoint having a bipartite branch.

If, on the other hand, $G_{2}$ is a star and $G_{1}$ contains a vertex with a bipartite branch, and (since $G$ is assumed connected) also contains an odd cycle, then the proof of part (b) of the Lemma implies the existence of a cutpoint in $G$.

\section{Unsolved problems.}

A. Connectivity. Weichsel [6] found the conditions for $G=G_{1} \wedge G_{2}$ to be connected. In the Theorem above, we derived a criterion for $G$ to be 2 -connected. When is $G$ n-connected? 
B. Digraphs. The conjunction of two digraphs was defined by McAndrew [5]; its connectedness categories were determined in Harary and Trauth [3]. What is the generalization of our Theorem to digraphs?

C. Factorization under conjunction. Some binary operations on graphs enjoy a unique factorization, but the conjunction does not. This is seen at once by the example $G=K_{2,2} \wedge P_{3} \cong K_{2,4} \wedge P_{2}$. There exist also examples of connected graphs with nonunique factorization. Dörfler [1] has shown how to obtain all "prime" factorizations from a given one. Which graphs have a unique conjunction-factorization?

\section{References}

[1] W. DöRfLER, Primfaktorzerlegung und Automorphismen des Kardinalproduktes von Graphen. Glasnik Mat. 9, 15-27 (1974).

[2] F. Harary, Graph Theory. Reading 1969.

[3] F. Harary and C. A. Trauth, Connectedness of products of two directed graphs. J. SIAM Appl. Math. 14, 250-254 (1966).

[4] F. HARARY and G. W. Wricox, Boolean operations on graphs. Math. Scand. 20, 41-51 (1967).

[5] M. H. McAndrew, On the product of directed graphs. Proc. Amer. Math. Soc. 14, 600-606 (1963).

[6] P. M. WeIChser, The Kronecker product of graphs. Proc. Amer. Math. Soc. 13, 47-52 (1962).

Eingegangen am 1. 12. 1976

Anschrift der Autoren:

Paul Hafner

Department of Mathematics

University of Auckland

Auckland, New Zealand
Frank Harary

Department of Mathematics

University of Michigan

Ann Arbor, Michigan, U.S.A. 